\title{
FACTORES DETRÁS DE LA RENUENCIA AL CONTROL VECTORIAL DEL DENGUE EN TRES DISTRITOS DEL NORTE DEL PERÚ
}

\author{
Helen Palma-Pinedo ${ }^{1, a}$, Rufino Cabrera ${ }^{1, b}$, Martín Yagui-Moscoso ${ }^{1, c}$
}

\begin{abstract}
RESUMEN
Objetivos. Identificar los factores detrás de la renuencia de la población a las acciones que conforman el control vectorial del dengue en tres distritos de la región Piura en el Perú. Materiales y métodos. El trabajo de campo se llevó a cabo en mayo de 2015 en los distritos de Cura Mori, Rinconada Llicuar y Sullana en la costa norte del Perú. La metodología empleada fue cualitativa con la finalidad de lograr el entendimiento e inclusión de la perspectiva de los actores involucrados en la problemática del dengue. Para ello, se empleó como técnicas la entrevista y la observación. Las entrevistas fueron abiertas en profundidad y se aplicaron de forma individual y grupal al equipo técnico responsable del control espacial y focal, así como a la población mediante visitas domiciliarias. Resultados. La renuencia estuvo basada en cuatro argumentos identificados: que la fumigación no es efectiva, la desconfianza frente al personal que realiza las intervenciones, que el horario de las actividades es inadecuado y que el larvicida empleado afectaba la calidad y sabor del agua. Se identificaron grupos renuentes heterogéneos constituidos por población de buena condición económica, residentes en áreas de alta peligrosidad o "zonas rojas", familias compuestas por algún personal de salud y viviendas con algún miembro enfermo o con discapacidad. Conclusiones. Los argumentos para la renuencia así como los grupos renuentes al control vectorial durante la epidemia de dengue en los distritos explorados son heterogéneos, por lo que las estrategias para su recuperación deben considerar estos aspectos.
\end{abstract}

Palabras clave: Dengue; Control de mosquitos; Fumigación; Entrevista; Factores sociológicos (fuente: DeCS BIREME).

\section{FACTORS BEHIND PEOPLE'S RELUCTANCE TOWARDS DENGUE VECTOR CONTROL ACTIONS IN THREE DISTRICTS IN NORTHERN PERU}

\begin{abstract}
Objectives. To identify factors behind a community's reluctance to dengue vector control efforts in three districts of the Piura region in Peru. Materials and methods. Fieldwork was conducted in May 2015 in the districts of Mori, Rinconada, Llicuar, and Sullana on the northern coast of Peru. Qualitative research methods were employed to understand varying perspectives of individuals involved in the dengue issue. Techniques such as interviewing and observation were used. Interviews included open-ended, in-depth questions. Individual interviews, focus groups, and home visits of community members were performed. Results. Reluctance was based on four arguments: 1 . Fumigation is ineffective; 2 . Personnel intervening are untrustworthy; 3 . The timing of activities is unsuitable; and 4 . The larvicide used affected water quality and taste. There was clear heterogeneity in reluctant groups: community members with high economic status, residents in high-risk or "red zone" areas, families with someone working as a healthcare professional, and houses with an ill or disabled member. Conclusions. The arguments behind reluctance as well as the composition of groups reluctant to vector control during dengue outbreaks in the districts examined were heterogeneous. Control strategies must take this heterogeneity into account.
\end{abstract}

Key words: Dengue; Mosquito control; Fumigation; Interview; Sociological Factors (source: MeSH NLM).

\section{INTRODUCCIÓN}

El dengue es una enfermedad viral endemoepidémica causada por cuatro serotipos del virus del dengue y trasmitida por el mosquito Aedes aegypti. Es la enfermedad viral transmitida por artrópodos más importante en términos de morbilidad y mortalidad $(1,2)$.
Su trasmisión ocurre en cerca de 140 países, principalmente de Asia, América y África. Se estiman 390 millones de casos nuevos en el mundo (96 millones de casos sintomáticos) y 25000 muertes cada año ${ }^{(1,3)}$. Las Américas vienen experimentando un incremento dramático del número de casos en la última década, estos representan el $14 \%$ de los casos sintomáticos a

\footnotetext{
Dirección General de Epidemiología, Ministerio de Salud. Lima, Perú.

Antropóloga; ${ }^{\mathrm{b}}$ biólogo; ${ }^{\mathrm{c}}$ médico

Recibido: 15-09-15 Aprobado: 09-03-16
}

Citar como: Palma-Pinedo H, Cabrera R, Yagui-Moscoso M. Factores detrás de la renuencia al control vectorial del dengue en tres distritos del norte del Perú. Rev Peru Med Exp Salud Publica. 2016;33(1):13-20. doi: 10.17843/rpmesp.2016.331.1900 
nivel mundial, habiéndose documentado la transmisión del dengue en casi todos los países, excepto Uruguay y Chile ${ }^{(4,5)}$.

Diversos factores han sido relacionados con la reemergencia de esta enfermedad, particularmente, el crecimiento poblacional y la urbanización no planificada, que han resultado en inadecuadas condiciones de vida, insuficiente abastecimiento de agua, así como la gestión incorrecta del alcantarillado y el manejo de los residuos sólidos. También han sido importantes el incremento de los viajes aéreos, la migración y el deterioro de los programas de prevención y control. La pobreza y las inequidades en salud han sido determinantes detrás de estos factores ${ }^{(6)}$

En el Perú, el control vectorial constituye el pilar de las actividades de prevención, el cual se realiza mediante el control de criaderos del Ae. aegypti a través de métodos físicos y químicos (control focal) y el control de los mosquitos adultos (control espacial) a partir de la fumigación de las viviendas en situaciones de brote ${ }^{(7,8)}$ para lo cual es necesaria la aceptación de las intervenciones por parte de la comunidad (9-13). Sin embargo, esto no siempre es posible debido a la renuencia de los pobladores a dichas actividades, o debido a la existencia de viviendas cerradas.

De acuerdo con la normatividad del Ministerio de Salud, la cobertura del control espacial y focal debería ser como mínimo de $95 \%$ de las viviendas, considerándose aceptable hasta un máximo de $5 \%$ de viviendas no inspeccionadas (cerradas, renuentes y deshabitadas). Una vivienda es definida como renuente cuando el jefe de familia presente al momento de la inspección no autoriza el ingreso del inspector; mientras que una vivienda cerrada es aquella que se encuentra así al momento de la inspección, o que no cuenta con la presencia de un adulto ${ }^{(14)}$. La elevada proporción de viviendas consideradas renuentes o cerradas constituye un problema para el control del dengue ${ }^{(15)}$.

En el 2014, la región Piura fue la segunda región con mayor número de casos de dengue, acumulando el 15,5\% de los casos notificados y $13,8 \%$ de las defunciones a nivel nacional ${ }^{(16)}$. El 2015 Piura presentó una epidemia, por lo que fue declarado en estado de emergencia sanitaria con el fin de desarrollar actividades en el marco del "Plan de acción de emergencia sanitaria región Piura-Año 2015". Hasta la semana epidemiológica 19 del 2015, el distrito de Tambo Grande había notificado 1409 casos de dengue que representaba el 16,3\% del total de casos reportados en la región. Por su parte, el distrito de Cura Mori había notificado 201 casos que representaba el $2,3 \%$ del total, y el distrito de Sullana notificó 1092 casos, que representaba el 12,6\%.
En el marco del plan antes mencionado se realizaron intervenciones de control vectorial consistentes en control focal con temefós y control espacial con malatión usando termonebulizadores; sin embargo, se obtuvo una alta tasa de viviendas renuentes y cerradas. A pesar que la falta de aceptabilidad es una conducta frecuente en las actividades de prevención y control, se han realizado pocos estudios orientados a observar las razones detrás de esta conducta. La mayoría de estudios se enfocan en evaluar los conocimientos, actitudes y prácticas de la población frente al dengue, lo cual constituye solo una arista del problema. En ese sentido, el presente estudio tuvo como objetivo el identificar las razones detrás de la renuencia de la población a las acciones que conforman el control vectorial en tres distritos de la región Piura.

\section{MATERIALES Y MÉTODOS}

El estudio se realizó del 27 de abril al 1 de mayo de 2015 en los distritos de Cura Mori, Rinconada Llicuar y Sullana, en la región Piura. Adicionalmente, se visitó Tambo Grande con el objetivo de dialogar con el personal que realizaba el control espacial y focal; sin embargo, en este distrito no se llegó a tener contacto con la población.

La metodología usada fue cualitativa, con la finalidad de lograr el entendimiento e inclusión de la perspectiva de los actores involucrados, principalmente de la población. Para ello, se aplicaron dos técnicas: la entrevista y la observación. Las entrevistas fueron abiertas en profundidad y se aplicaron tanto de forma individual como a partir de grupos focales a miembros del equipo técnico responsable del control vectorial, personal que realiza el control espacial y focal, así como a la población general. Todas las entrevistas fueron grabadas en audio previo consentimiento y fueron conducidas por uno de los autores (HP) con el apoyo de un técnico en el tema (RC) siguiendo la guía de preguntas previamente diseñada.

Con el grupo de trabajadores de salud, además de las entrevistas realizadas a diez profesionales de la DIRESA y personal de los distritos seleccionados, se tuvo la oportunidad de participar en reuniones de coordinación entre funcionarios y especialistas de los componentes involucrados (Epidemiología, Salud Ambiental, Promoción de la Salud, Comunicaciones) para conocer las actividades y limitaciones en la prevención, vigilancia y control del dengue. Los grupos focales se realizaron con inspectores que realizan la vigilancia y el control focal en Tambo Grande $(n=12)$, Cura Mori $(n=15)$ y personal del control espacial $(n=12)$ de la Subregión de Salud de Luciano Castillo Colonna.

Para explorar la percepción de la población, se visitaron los domicilios para mantener un diálogo directo y observar las formas de almacenamiento del agua, así como 
registrar sus estrategias personales para la prevención del dengue. En este caso, se buscó tomar contacto tanto con las personas que presentaron aceptación a la acciones, como aquellas que fueron renuentes, con el objetivo de contrastar las diferentes posiciones.

Además, se acompañó a una jornada, en campo, de tratamiento espacial e inspección domiciliaria en el distrito de Cura Mori con el fin de registrar las características de este proceso, sus dificultades y, sobre todo, recoger las percepciones de la población acerca de la labor realizada en ese momento. De esta manera, se pudo observar el trabajo desde su organización inicial en las primeras horas del día hasta su desenvolvimiento en campo y las interacciones con la población.

Las dimensiones planteadas para la recolección y análisis de la información fueron:

- Percepciones sobre los factores detrás de la renuencia de la población a las acciones del control vectorial: para este caso se buscó conjugar los puntos de vista de los funcionarios y personal involucrado con las razones vertidas por la población, priorizando estas últimas.

- Percepciones de la población sobre las acciones que conforman el control vectorial: se buscó recoger la percepción de la población sobre dicho trabajo, así como de la utilidad que estas tienen desde su punto de vista para controlar el dengue.

A partir de estas dimensiones se plantearon preguntas siguiendo las técnicas antes descritas y que fueron punto de partida para el análisis posterior. Las entrevistas fueron transcritas y codificadas tomando en cuenta las dimensiones antes mencionadas. Este estudio fue realizado en el contexto de la epidemia de Piura, por ello, no pasó por el comité de ética. Todos los entrevistados participaron voluntariamente $y$ fueron informados previamente de los objetivos del estudio. Finalmente, se garantizó la protección de la identidad de los participantes y las preguntas abordaron las actividades rutinarias de prevención y control del dengue.

\section{RESULTADOS}

\section{CARACTERÍSTICAS DE LOS ENTREVISTADOS}

Los inspectores de Tambo Grande fueron, principalmente, mujeres; su promedio de edad fue de 33,1 años (rango: 25-43 años) y el promedio del tiempo de experiencia 2,3 años (rango: 0,8-5 años). Los inspectores entrevistados de Cura Mori fueron, en su mayoría, mujeres, el promedio de edad fue 38,2 años (rango: 27-50 años) y el tiempo promedio de experiencia fue 8,2 años (rango: 0,8-15 años).
El personal de control espacial (fumigador) estuvo compuesto por varones, cuyas edades estaban entre los 35 a 50 años y la experiencia varió entre 2 a 25 años.

\section{ENTENDIENDO LA RENUENCIA AL CONTROL VECTORIAL}

Se observó que en los casos de falta de aceptabilidad analizados, muchos habían accedido a que el personal ingresara a sus viviendas una primera vez, pero en la segunda o tercera visita se dio la renuencia. Se encontró que esto respondía principalmente a dos razones. La primera es que en el caso de la fumigación algunos aceptaban la primera intervención con la curiosidad de comprobar sus resultados, pero luego, al no ver satisfechas sus expectativas optaban por negarse a una siguiente. La segunda razón, fue la falta de disponibilidad en ese momento para hacer ingresar al personal.

Por otro lado, se identificó el hecho de que una familia no sea renuente no quiere decir que vaya a cumplir con las indicaciones dadas. Esto es reconocido por el personal de salud, quienes denominaban a estos casos como una "aceptación hipócrita", que se observaba, por ejemplo, en el hecho de aceptar el temefos y luego botarlo.

En palabras del personal, la renuencia es un problema que se presenta más en las familias de mejor condición económica, mientras que la aceptación suele ser mayor en aquellas que residen en zonas rurales o que presentan condiciones menos favorables. La renuencia también es alta en las llamadas "zonas rojas", es decir, que presentan un alto índice de peligrosidad, en las cuales no se permite el ingreso de personas extrañas para evitar quedar al descubierto ante las autoridades. Otro grupo mencionado como renuente en más de una ocasión fue el de familias compuestas por algún personal de salud que bajo el argumento de que tienen un mayor conocimiento sobre el dengue consideraban que no era necesario que se haga una intervención en su vivienda. Un último grupo fue aquél en el que existe un miembro de la familia con alguna enfermedad o discapacidad.

\section{ARGUMENTOS DE LA POBLACIÓN QUE JUSTI- FICAN LA RENUENCIA}

Los argumentos en contra del control espacial y focal, al igual que los grupos renuentes, no son los mismos en todos los casos. A partir del diálogo directo con la población se obtuvo cuatro argumentos que, desde su punto de vista, justifican la renuencia.

\section{Argumento 1: "La fumigación no sirve"}

Se encontró que un amplio grupo de personas, tanto renuentes como con aceptación al control espacial y con experiencia previa en el tratamiento espacial, consideraba 
que esta es una acción que no da resultados. Desde su perspectiva, esto se fundamentaba, principalmente, en cinco hechos (Figura 1). El primero es que la cantidad de zancudos no se reduce y que estos se retiran el tiempo que dura la fumigación, pero luego regresan. El segundo hecho es que las horas en las que se realiza la fumigación es vista como inadecuada, porque los zancudos se encuentran o muy temprano en la mañana o ya llegada la tarde; sin embargo, la fumigación (al menos en los casos observados) se realizaba entre las 07.00 y $13.00 \mathrm{~h}$ lo que era considerado ineficiente, porque el vector no se encontraba en esas horas. En tercer lugar, está el hecho que las viviendas sean abiertas, lo que hace pensar que el producto es desaprovechado lo que reduce su efectividad. En cuarto lugar, consideraban que no tenía ningún sentido fumigar las viviendas ya que el zancudo viene de los campos de cultivo, que en estos casos no son muy apartados y que, por tanto, se debería fumigar también en los exteriores. En quinto lugar, se considera que el tener viviendas vecinas abandonadas $u$ otros reservorios hace que se concluya que la fumigación de su vivienda no sirve.

"El efecto de la fumigación dura poco, mata la primera vez, pero luego no".

"El animal viene del campo, es por las puras que fumiguen solo en la casa".

"La fumigación no hace efecto para nada. Al contrario, vienen más moscas y zancudos. A mí me fumigaron las tres veces y nada, igualito, se llena de humo todo, pero cuando abrimos nuestra casa igualito es".

Pobladores de Rinconada Llicuar

"Pienso que se debería fumigar a los alrededores, porque el zancudo está metido en los montes".

"Mi negocio ya lo han fumigado antes, lo llenan de humo y al final no sirve para nada. Para mí es como si no hicieran nada. Acá sencillamente no le dejé entrar ahora porque estoy en mi negocio y me incomoda. Pienso que es por las puras".

\section{Pobladores de Cura Mori}

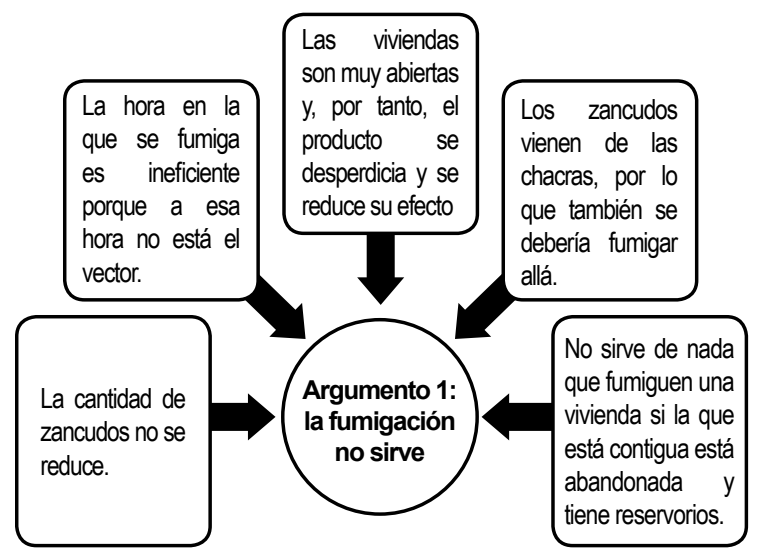

Figura 1. Razones detrás del primer argumento identificado en contra del control espacial

\section{Argumento 2: "El personal me da desconfianza"}

En este argumento, el tema de fondo es la desconfianza, la cual se presenta en circunstancias en las que la información previa a la población ha sido insuficiente o cuando los fumigadores no son acompañados de otro personal que vaya alertando a los vecinos de su llegada (Figura 2). En Tambo Grande, por ejemplo, se encontró que en esa ocasión los fumigadores visitaron solos las viviendas, lo cual generó rechazo en la población, a lo que sumaba el hecho que este grupo provenía de Sullana por lo tanto, no eran conocidos. Este es un argumento también reconocido por el personal. Anteriormente, con el uso de la cipermetrina, una persona de la vivienda era invitada a acompañar al fumigador mientras este hacía su labor como una estrategia para aumentar la confianza. Pero, ahora que el insecticida es un organofosforado esto ya no es posible, lo cual representa una barrera.

Por otro lado, se han recibido acusaciones de robo de objetos personales de la población hacia el personal, sobre todo el del control espacial, pero hasta ese momento no había una evidencia de su veracidad.

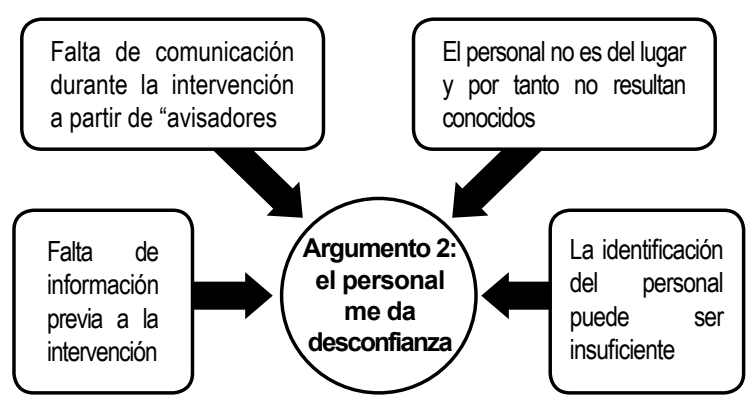

Figura 2. Razones para la desconfianza hacia el personal del control vectorial

\section{Argumento 3: "El personal viene a mala hora"}

Uno de los principales problemas del control vectorial, especialmente del control espacial, es que sus horarios no se ajustan a las rutinas de las familias. El trabajo se suele desarrollar en días útiles y generalmente por las mañanas, afectando así las actividades de las familias (Figuras 3). Durante el acompañamiento que se hizo, se observó que se generaba incomodidad en varios casos ya que las madres estaban preparando a sus hijos para el colegio, estaban desayunando, y algunas empezaban a cocinar para el almuerzo.

"Yo no dejé fumigar mi casa la primera vez porque estaba haciendo el afrecho para mi chicha, y la otra vez estaba cocinando.

Entrevistador: ¿Pero le avisaron que irían a esa hora a fumigar?

Sí, pero qué voy a hacer, igual tengo que cocinar todos los días. La última vez que vinieron sí dejé que entren porque todavía no estaba cocinando".

Pobladora de Rinconada Llicuar 
Un patrón en todos los lugares visitados fue que las viviendas con más renuencia eran aquellas donde se desarrollaba algún tipo de negocio como juguerías, bodegas, venta de chicha, etc. Este tipo de negocios tienen horas de mayor actividad en las mañanas, y en el caso de la chicha es la hora en la que esta se prepara. En estos casos, los propietarios no estaban dispuestos a afectar su economía por la fumigación, pero sí estaban dispuestos a dejar pasar al personal en horas que los afecte menos.

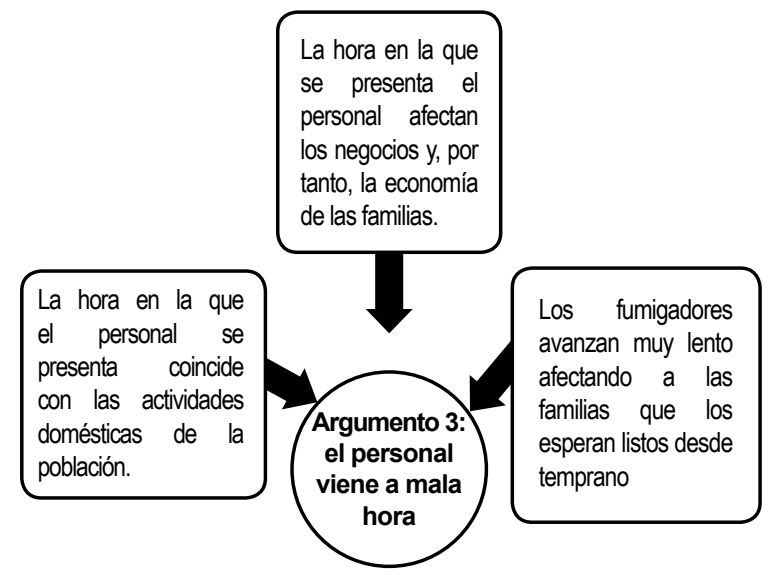

Figura 3. Razones detrás del tercer argumento identificado

\section{Argumento 4: "El temefós deja al agua un olor y sabor desagradables y además la ensucia"}

Este es un argumento que afecta específicamente al control focal. Se identificó que un sector de la población presentaba rechazo a su uso por el cambio que este generaría en las características del agua, la cual tenía un uso doméstico y para el consumo humano. En el caso de Tambo Grande, esto coincidía con el cambio en la marca utilizada; no obstante, en los lugares donde sí fue posible dialogar con la población el número de personas que dieron este argumento no fue mayoritario. Las inspectoras entrevistadas de Tambo Grande reconocieron que el temefós utilizado en ese momento sí cambiaba el olor, a diferencia del anterior, lo que en sus palabras se hacía bastante notorio al destapar depósitos que habían permanecido cerrados por varias horas.

En cuanto a su rápida eliminación, que se sabe es una gran preocupación para el personal de salud, se detectaron casos en ámbitos donde el recambio de agua de los envases es muy frecuente. Así, en el asentamiento humano Villa Primavera se encontraron viviendas en las que se manifestaba que estas habían sido eliminadas al día siguiente, cuando se hizo el cambio de agua en sus depósitos.

\section{DISCUSIÓN}

En este estudio se identificaron varios nudos críticos desde la percepción de los trabajadores de salud (fumigadores e inspectores domiciliarios) y la población (renuentes a las actividades de control y aquellos que aceptaron las intervenciones). Por un lado, parte de la población consideraba que la fumigación no era efectiva para controlar el Ae. aegypti o que solo lo hacía por un período corto, lo cual también ha sido descrito en estudios realizados en Costa Rica y Cuba en los que los pobladores no perciben los beneficios de dicha actividad y consideran que incluso podría ser peligrosa para la salud (17). Por otro lado, un estudio cualitativo realizado en informantes claves y en 40 personas con 39,5 años, en promedio de edad, de los cuales, la mitad fueron hombres, identificaron que existe una excesiva confianza en el control del dengue a través de la fumigación (18). Es decir, que el control del dengue es asociado con la fumigación, lo cual se evidencia en esta investigación.

La duración del efecto letal de los aerosoles del insecticida contra los mosquitos en el ambiente es muy corto ${ }^{(19)}$, lo cual es percibido muy bien por la población. La escasa información que esta recibe sobre las bondades y limitaciones de la fumigación puede contribuir a la renuencia y al impacto de esta intervención. La población también debe conocer mejor la biología del vector y el comportamiento de los adultos hembras de Ae. Aegypti, para diferenciarlo de otros mosquitos no involucrados en la transmisión.

Otro de los aspectos clave en las intervenciones es el horario, que se debe más a razones operativas del equipo de salud. La intervención debe estar en relación con la mayor actividad de picadura del vector ${ }^{(19)}$ para que tenga mayor impacto en su reducción y en concordancia con la Norma Técnica de Vigilancia y Control (14). Aquí hay un factor crítico, que es contar con medios de transporte adecuados para el traslado del personal y sus equipos a las zonas de intervención. Los equipos de gestión deben considerar este aspecto en la planificación de los costos de intervención para mejorar la aceptabilidad de la población y el impacto de la intervención.

Las viviendas abandonadas y las renuentes también cumplen un papel muy importante en el control de la transmisión, puntos que han sido reconocidos por la población. El Ministerio de Salud ha normado la intervención de las viviendas cuyos jefes de familia se resisten a la intervención mediante la participación de la Fiscalía y la Policía Nacional del Perú para mejorar la cobertura de control vectorial (20). En estos casos se debe gestionar con los responsables de promoción de la salud para que se sensibilice acerca de la necesidad 
de efectuar las acciones de control del brote, o que la autoridad competente gestione la forma de lograr que estas viviendas sean inspeccionadas y tratadas, o que se programe la recuperación de tales viviendas en diferentes horarios, como se recomienda ${ }^{(14)}$.

Por otro lado, desde la perspectiva de los fumigadores, las condiciones en que laboraban bajo el intenso calor, las medidas de protección, la deshidratación que sufren y el monitoreo del nivel de toxicidad, son aspectos que el equipo de salud debería vigilar en forma permanente, así como, buscar estrategias para mejorar el rendimiento del personal y una mayor aceptabilidad de la población hacia la fumigación.

Con relación al argumento de la desconfianza, la solución debería incluir acciones como realizar una adecuada comunicación a lo largo de las actividades de prevención y el control vectorial, como el acompañamiento por parte del personal de salud de la zona durante la intervención en las viviendas. El argumento de la desconfianza es algo que fácilmente puede presentarse en la mayoría de distritos. En este caso, el estudio se desarrolló en espacios de tipo urbano marginal, pero es de esperar que la desconfianza también esté presente, o que incluso se incremente, en la población de zonas de mayor poder adquisitivo. Esto coincide con los resultados de un estudio realizado en Sao Paulo-Brasil sobre las dificultades para la aceptabilidad de la población al programa de control de dengue, en donde se encuentra que la falta de información y la desconfianza causada por los índices de violencia en la zona es una de las barreras para realizar dicho trabajo ${ }^{(21)}$

Otra barrera importante es que la población no entiende, o no acepta, la función de los larvicidas en la prevención y control del dengue ${ }^{(12)}$. A esto se suma lo observado en este estudio respecto de que el larvicida empleado en Piura cambiaría el sabor, apariencia y olor del agua, situación que ya ha sido reportada en otros países como Cuba y Tailandia ${ }^{(19,24)}$. La recomendación para abordar este argumento es confirmar si el nuevo producto utilizado realmente ocasiona estos cambios en el agua y cuánto es la tasa de rechazo de la población por esta razón. Consideramos que en la compra de larvicidas se debe tener en cuenta no solo la efectividad y el costo, sino también las preferencias de la población pudiéndose optar por otro producto que reduzca dichos efectos para mejorar el impacto del control.

Esto concuerda con un estudio realizado en las provincias de Santiago de Cuba y Guantánamo en un horizonte temporal de 7 años en el marco de la implementación de investigaciones operacionales sobre el diseño, implementación y evaluación de estrategias comunitarias insertadas en el Programa Cubano de Control de
Ae. aegypti para la prevención del dengue ${ }^{(22)}$. En dicho estudio se encontró que el 33,5 \% de la población era renuente a las actividades de control de vectores porque consideraban que el temefos daba mal sabor al agua y no lograba eliminar los huevos del mosquito, las horas de visita de los trabajadores de control de vectores era considerada inadecuada por la población y por desconfianza en las intervenciones realizadas por el personal de salud (básicamente en la calidad del trabajo). Si bien es cierto que existen muy pocas investigaciones publicadas de este tipo, es posible que este contexto sea frecuente en otros países de América.

Por otro lado, se identificaron grupos renuentes constituidos, principalmente, por población de buena condición económica y que residen en zonas céntricas, población que habita en áreas de alta peligrosidad o "zonas rojas", viviendas con personal de salud (que argumentan mayor conocimiento acerca del dengue) y viviendas con algún miembro enfermo o con discapacidad. Los resultados evidencian que las estrategias para la recuperación de las viviendas renuentes no pueden ser las mismas ya que tanto los grupos renuentes como los argumentos y razones detrás de esta conducta son heterogéneos, por lo que no es posible aplicar un único criterio para todos los casos; sin embargo, podría obtenerse mayor aceptabilidad de la población en la medida que se mejore las estrategias de comunicación y promoción. Una propuesta al respecto fue la intervención de un fiscal para forzar el ingreso a las viviendas. Esta debe ser una medida pensada para sectores críticos o con historia de baja cobertura de control (20); como se puede observar, los grupos que conforman el sector renuente no son homogéneos ni presentan los mismos motivos, y existe el riesgo de tener un resultado contraproducente.

En general, se comprobó que la población está bastante informada sobre el dengue, lo cual, suele observarse en áreas endemoepidémicas ${ }^{(21,22,25)}$, lo que hace falta es informar más y mejor acerca de las acciones del control vectorial. Esto debe extenderse durante todo el tiempo que duren las actividades, tanto en escenarios no epidémicos como epidémicos ${ }^{(20)}$. Con ello se contribuirá a fortalecer la confianza de la población hacia el personal y lo que se hace.

Este estudio presenta la limitación de haber sido realizado solo en el ámbito de tres distritos en un contexto epidémico; no obstante, consideramos que dado que las acciones del control vectorial son las mismas y son ejecutadas con recursos humanos y materiales similares, los resultados no serían discrepantes con los presentados en otros distritos de Piura con características semejantes. Por otro lado, si bien se tomó en cuenta tanto a la población renuente como la que presentó aceptación al control vectorial, 
ello permitió evidenciar que las viviendas renuentes no son siempre las mismas y que las viviendas que fueron intervenidas una vez pueden volverse renuentes si no se toman en cuenta los aspectos que fueron identificados.

Se recomienda que se realicen más estudios con este enfoque para conocer mejor los determinantes asociados con la escasa aceptabilidad de la población.
Fuentes de financiamiento: este estudio fue financiado por la Dirección General de Epidemiología.

Conflictos de interés: los autores declaran no tener conflictos de interés.

Contribuciones de autoría: HP participó en la concepción del estudio, recolección, análisis e interpretación de los datos, y redacción del manuscrito. $\mathrm{RC}$ participó en la obtención de datos, hizo la revisión crítica del artículo, hizo aportes a su redacción y dio su aprobación final. MY participó en la concepción del artículo, la revisión crítica y su aprobación final.

\section{REFERENCIAS BIBLIOGRÁFICAS}

1. Hermann LL, Gupta SB, Manoff SB, Kalayanarooj S, Gibbons RV, Coller BA. Advances in the understanding, management, and prevention of dengue. J Clin Virol. 2015;64(3):1539. doi: 10.1016/j.jcv.2014.08.031.

2. Guzman MG, Kouri G. Dengue and dengue hemorrhagic fever in the Americas: lessons and challenges. J Clin Virol. 2003;27(1):1-13. doi:10.1016/ S1386-6532(03)00010-6.

3. Bhatt S, Gething PW, Brady OJ, Messina JP, Farlow AW, Moyes CL, etal. The global distribution and burden of dengue. Nature 2013;496(7446):5047. doi: $10.1038 /$ nature 12060 .

4. Organización Panamericana de la Salud (OPS). Situación epidemiológica del dengue [Internet]. Bucaramanga: OPS/OMS; 2014 [citado el 12 de julio de 2015]. Disponible en: http://www.paho.org/hq/index. php?option $=$ com_docman $\&$ task $=$ doc download\&Itemid=\&gid=26802\&lang=es

5. San Martín JL, Brathwaite O, Zambrano B, Solórzano JO, Bouckenooghe A, Dayan GH, et al. The epidemiology of dengue in the americas over the last three decades: a worrisome reality. Am J Trop Med Hyg. 2010;82(1):128-35. doi: 10.4269/ajtmh.2010.09-0346.

6. Guzman MG, Kouri G. Dengue and dengue hemorrhagic fever in the Americas: lessons and challenges. J Clin Virol. 2003;27(1):1-13. doi: 10.1016/S1386-6532(03)00010-6

7. San Martín JL, Brathwaite-Dick O. La estrategia de gestión integrada para la prevención y control del dengue en la región de las Américas. Rev Panam Salud Publica. 2007; 21(1):55-63. doi: 10.1590/S1020-49892007000100011.

8. Achee NL, Gould F, Perkins TA, Reiner RCJr, Morrison AC, Ritchie SA, et al. A
Critical Assessment of Vector Control for Dengue Prevention. PLoS Negl Trop Dis. 2015;9(5):e0003655. doi: 10.1371/journal.pntd.0003655.

9. Toledo-Romani ME, Baly-Gil A, Ceballos-Ursula E, Boelaert M, Van der Stuyft P. Participación comunitaria en la prevención del dengue: un abordaje desde la perspectiva de los diferentes actores sociales. Salud Publica Mex. 2006;48(1):39-44. doi: 10.1590/ S0036-36342006000100007.

10. Castro M, Sanchez L, Pérez D, Carbonell N, Lefèvre P, Vanlerberghe $\mathrm{V}$, et al. A community empowerment strategy embedded in a routine dengue vector control programme: a cluster randomised controlled trial. Trans $\mathrm{R}$ Soc Trop Med Hyg 2012;106(5):31521. doi: 10.1016/j.trstmh.2012.01.013.

11. Asian Development Bank; World Health Organization. Managing regional public goods for health: Community-based dengue vector control [internet]. Mandaluyong City, Philippines: Asian Development Bank; 2013 [citado el 11 de junio de 2015]. Disponible en: http://www.adb.org/ sites/default/files/publication/30167/ community-based-dengue-vectorcontrol.pdf

12. Sommerfeld J, Kroeger A. Innovative community-based vector control interventions for improved dengue and Chagas disease prevention in Latin America: introduction to the special issue. Trans R Soc Trop Med Hyg. 2015;109(2):85-8. doi: 10.1093/ trstmh/tru176. doi: 10.1093/trstmh/ tru176.

13. Avila Montes GA, Ponce C, Ponce E, Martínez Hernández M, Flores M. Insecticidal paint and fumigant canisters for Chagas' disease control: community acceptance in Honduras. Rev Panam
Salud Publica.1999;6(5):311-20. doi: 10.1590/S1020-49891999001000003.

14. Perú, Ministerio de Salud. Dirección General de Salud Ambiental. Norma Técnica de Salud para la implementación de la vigilancia y control del Aedes aegypti, vector del dengue y la fiebre chikungunya y la prevención del ingreso del Aedes albopictus en el territorio nacional. Lima: Dirección General de Salud Ambiental - Ministerio de Salud; 2015.

15. Vilcarromero S, Casanova W, Ampuero JS, Ramal-Asayag C, Siles C, Díaz G, et al. Lecciones aprendidas en el control de Aedes aegypti para afrontar el dengue y la emergencia de chikungunya en Iquitos, Perú. Rev Peru Med Exp Salud Publica. 2015;32(1):172-8. doi: 10.17843/rpmesp.2015.321.1590.

16. Perú, Ministerio de Salud, Dirección General de Epidemiología. Sala situacional para el Análisis de Situación de Salud 2014 [Internet]. Lima: Minsa [citado el 4 de enero de 2015]. Disponible en: http://www.dge.gob. $\mathrm{pe} /$ portal/index.php?option $=\mathrm{com}$ content $\&$ view $=$ article $\&$ id $=447$

17. Pérez-Guerra CL, ZielinskiGutierrez E, Vargas-Torres D, Clark GG. Community beliefs and practices about dengue in Puerto Rico. Rev Panam Salud Publica. 2009;25(3):218-26. doi: 10.1590/ S1020-49892009000300005.

18. Torres López TM, Guerrero Cordero JL, Salazar Estrada JG. Dimensiones culturales del dengue que favorecen o dificultan su prevención en México. Rev Panam Salud Publica. 2012;31(3):197-203. 10.1590/ S1020-49892012000300003. 
19. Bonds JA. Ultra-low-volume space sprays in mosquito control: a critical review. Med Vet Entomol. 2012 Jun;26(2):121-30. doi: 10.1111/j.1365-2915.2011.00992.x.

20. Perú, Ministerio de Salud. Acciones de promoción de la salud para la prevención del dengue a través del abordaje de los determinantes sociales de la salud. Lima: Dirección General de Promoción de la Salud, MINSA; 2014.

21. Chiaravalloti Neto F, Baglini V, Cesarino MB, Favaro EA, Mondini A, Ferreira AC, et al. O Programa de Controle do Dengue em São José do Rio Preto, São Paulo, Brasil: dificuldades para a atuação dos agentes e adesão da população. Cad. Saúde Pública. 2007; 23(7):1656-1664. doi: 10.1590/ S0102-311X2007000700017.
22. Toledo-Romani ME. Ensayos comunitarios para la prevención del dengue: De la investigación formativa a la práctica. [tesis doctoral]. La Habana: Instituto de Medicina Tropical "Pedro Kourí"; 2009. Disponible en: http:// tesis.repo.sld.cu/67/2/mariae_toledo. pdf.

23. Pérez-Guerra CL, Seda H, GarcíaRivera EJ, Clark GG. Knowledge and attitudes in Puerto Rico concerning dengue prevention. Rev Panam Salud Publica. 2005;17(4):243-53. doi: 10.1590/S1020-49892005000400005.

24. Wong LP, Shakir SM, Atefi N, AbuBakar S. Factors affecting dengue prevention practices: nationwide survey of the Malaysian public. PLoS One. 2015;10(4): e0122890. doi: 10.1371/journal.pone.0122890.
25. Flynn A. A study exploring the knowledge, attitudes and practices of young people regarding dengue fever and the extent of community involvement in vector control of the disease in Trinidad and Tobago. West Indian Med J. 2012;61(6):615-8.

Correspondencia: Helen Palma Pinedo Dirección: Daniel Olaechea 199, Jesús Maria, Lima Perú.

Teléfono: (511) 631-4500

Correo electrónico:helenpalmapinedo@ hotmail.com

\section{http://twitter.com/rpmesp}

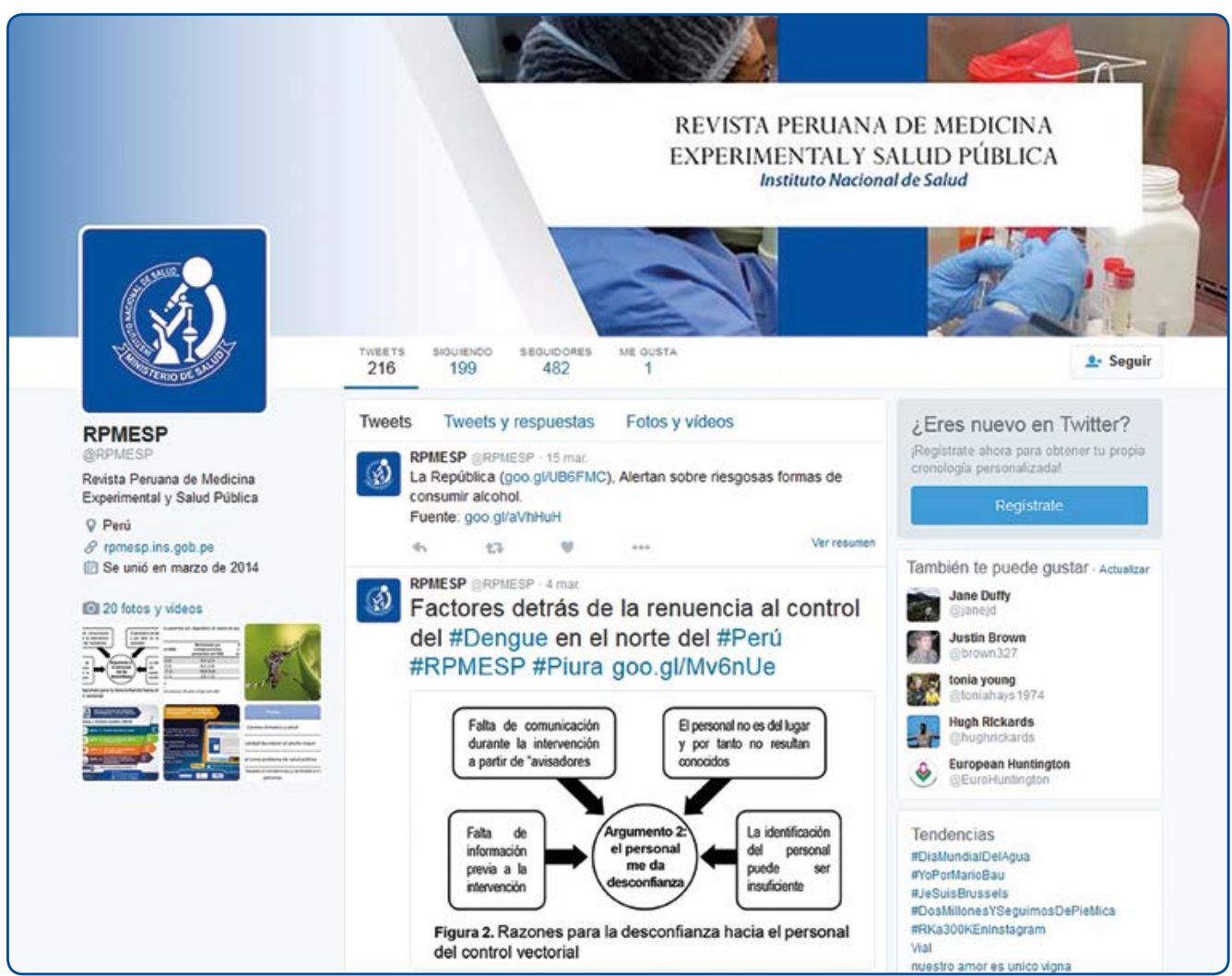

